\title{
EL PRINCIPIO DE PRECAUCIÓN EN EL PLAN NACIONAL DE SALUD PÚBLICA DE COLOMBIA: ¿AUSENCIA O GRAN MAL?
}

\author{
Germán Agudelo Montoya MD. \\ Profesor Universidad de Caldas \\ german.agudelo@ucaldas.edu.co \\ José Fernando López Mejía MD. \\ Auditor Hospital de Caldas \\ jofelome@une.net.co
}

Manizales, 2009-05-06 (Rev. 2009-06-07)

\section{RESUMEN}

Este artículo resume el informe final de la investigación que lleva como título: "Apropiación del Principio de Precaución en las políticas públicas sanitarias: caso Plan Nacional de Salud Pública de Colombia", el cual está centrado en el estudio del Principio de Precaución y su incidencia en las políticas públicas sanitarias de la legislación colombiana. La investigación se centró en la revisión tanto de la historia de tal principio en la legislación internacional, fundamentalmente en las convenciones sobre medio ambiente, como en la exploración conceptual que se ha hecho del principio y su relación con la salud pública, especialmente en lo que refiere al Plan Nacional de Salud Pública, determinando la apropiación o no del principio en tal política. Aparece en escena y como correlato investigativo una apertura conceptual del concepto de salud y del concepto de riesgo, los cuales se involucran en la discusión para poder determinar la efectiva incidencia de la precaución en la legislación colombiana. La pretensión de este artículo es, entonces, mostrar de manera sintética cada uno de los puntos a los que hace referencia la investigación misma, esto es, los orígenes y el contexto del Principio de Precaución, las aproximaciones conceptuales que se han hecho acerca de él, su relación específica con la legislación colombiana, los vínculos que éste tiene dentro del complejo concepto de la salud pública y su correspondencia particular en el Plan Nacional de Salud Pública en Colombia. El análisis realizado permitió identificar algunos criterios útiles para determinar el grado de apropiación del Principio de Precaución de una política pública sanitaria. En este sentido se encontró que en el país sí existe legislación que propende por la inclusión del Principio de Precaución en las normas, pero el actual Plan Nacional de Salud Pública no lo contempla ni explícita ni implícitamente.

\section{PALABRAS CLAVE}

Principio de Precaución, Plan Nacional de Salud Pública, Medio Ambiente, Daño, Riesgo, Incertidumbre, Salud, Colombia.

\section{THE PRECAUTIONARY PRINCIPLE IN THE COLOMBIAN NATIONAL PUBLIC HEALTH PLAN: ¿ABSENCE OR IMPIDEMENT?}

\begin{abstract}
This article summarizes the final report of the research entitled "Apropiación del Principio de Precaución en las políticas públicas sanitarias: caso Plan Nacional de Salud Pública de Colombia" which is focused on the study of the Precautionary Principle and its incidence on public health policy of the Colombian legislation. The research covered the historical review of such principle in international legislation, especially on the environmental conventions. It also studied the conceptual exploration that has been carried out on the principle and its relationship with public health, particularly in regards to the Colombian National Public Health Plan, determining the principle's acquisition or not in such plan. As parallel research points, the concepts of health and risk are treated, which come into play
\end{abstract}


in this discussion in order to determine the effective incidence of precaution in the Colombian legislation. What this article intends is to show, in a synthetic way, each of the points to which the research itself makes reference, the origins and context of the Precautionary Principle; the conceptual approaches that have been used for its study, its specific relationship with Colombian legislation; the links that this principle has inside the complex concept of Public Health; and, its particular correspondence in the National Public Health Plan in Colombia. The analysis identified some useful criteria for determining the acquisition level of the Precautionary Principle of a public health policy in Colombia. In this sense, the results showed that the country possesses legislation that favors the inclusion of the Precautionary Principle within the norms, but the current National Public Health Plan does not contemplate it, neither implicitly nor explicitly.

\section{KEY WORDS}

Precautionary Principle, Colombian Public Health National Plan, Environment, Damage, Risk, Uncertainty, Health, Colombia.

\section{INTRODUCCIÓN}

Las contemporáneas preocupaciones sobre el medio ambiente y su incidencia en la salud humana implican el estudio del Principio de Precaución como política vinculante de la salud pública, de las políticas públicas ambientales y sanitarias, de los compromisos de los gobiernos nacionales y los acatamientos de reglamentaciones internacionales.

Las políticas públicas sanitarias asumen los avances científicos en su diseño, elaboración y aplicación, pero la ciencia, con sus métodos, sólo avanza al demostrar relaciones causales y certezas en el marco de intervalos de confianza que permiten obviar el riesgo del azar, pero en la mayoría de los casos, las posibles futuras consecuencias dañosas o no hacia la salud humana y hacia el medio ambiente de una determinada política o decisión no son determinadas con igual certeza por parte de esa ciencia, en tanto se requiere una predicción sin hechos mesurables o la realización de estudios prolongados. El saber ambiental, interdisciplinario desde sus fundamentos, ha llegado al establecimiento del Principio de Precaución que, como herramienta conceptual y práctica, puede facilitar el abordaje del estudio de las consecuencias de las políticas públicas en un contexto de incertidumbre.

El propósito de este artículo es referir algunas de las consideraciones que deben tenerse en cuenta para la investigación sobre las normativas nacionales e internacionales acerca de la salud pública y el medio ambiente, enlazadas con la apertura del concepto de salud, con la perspectiva de riesgo, los daños graves o irreversibles y la carencia de certeza científica absoluta. Para ello se indaga en las normativas que en Colombia refieren al Principio de Precaución, a las declaraciones y legislaciones internacionales al respecto, para luego ingresar al estudio del Plan Nacional de Salud Pública en Colombia.

\section{METODOLOGÍA}

La investigación realizada ${ }^{1}$ fue de tipo cualitativo y se centró en el análisis documental y en la emergencia de categorías derivadas del análisis de la estructura de las políticas públicas sanitarias de Colombia. Para el abordaje inicial se tuvo en cuenta, además de la identificación de la política, el objeto, el grado de dependencia del Estado, su inmediatez, el tipo de financiación prevista, el alcance y mensurabilidad del impacto, la existencia de indicadores de seguimiento, la claridad de la redacción del articulado y la interrelación con otras políticas públicas nacionales. Algunas de las categorías de análisis que emergieron de la revisión teórica y del análisis de las políticas públicas sanitarias fueron: concepto de salud, concepto de riesgo, concepto de medio ambiente, tipo de participación, certidumbre científica, tipificación de los riesgos, definición del riesgo, revisabilidad, generaciones futuras y sustentabilidad. 


\section{RESULTADOS Y DISCUSIÓN}

El Principio de Precaución nace de la imposibilidad, en todos los casos, de la certidumbre, esto es, de la falta de certeza científica absoluta. Si no es posible medir o registrar las consecuencias de una actividad de cualquier índole sobre la base de los riesgos probables graves o irreversibles, es el Principio de Precaución, como instrumento, quien debe estar presente en los órganos decisores y en la gestión de riesgos. Por lo tanto, el Principio de Precaución estaría inserto en "toda norma que establece una pauta a seguir ante la falta de certeza científica en la gestión de un riesgo ambiental o para la salud pública de cierta entidad, independientemente del contenido exacto que se haya dado a la propuesta normativa"2. El Principio de Precaución, así mismo, define la actitud que debe observar quien toma una decisión relativa a una actividad que conlleva a riesgo.

Rastrear el Principio de Precaución, por lo tanto, implica identificar las discusiones en las que éste ha sido mencionado y las características de esos debates, es decir, sus argumentos, sus premisas y sus contextos. Así mismo, implica identificar las normativas internacionales que consolidaron y pusieron en marcha este Principio en sus políticas constitutivas. Podríamos decir que este Principio dio su asomo en las convenciones internacionales sobre medio ambiente, justo cuando temáticas tales como la contaminación, el medio ambiente y la salud humana se introdujeron en las preocupaciones políticas, económicas y éticas de los países miembros de la Organización de las Naciones Unidas. Si bien la Conferencia sobre Medio Ambiente Humano de la ONU, en 1972, vislumbró de manera tímida lo que más tarde sería el Principio de Precaución, fue la República Federal Alemana, en 1974, la que específicamente y como ley estatal hace mención puntual al Vorsorgeprinzip, recalcando que "la política ambiental no se agota en la defensa contra peligros amenazantes y la reparación de daños ya acaecidos. Una política ambiental precautoria (vorsongende Umweltpolitik) exige, más allá de eso, que los fundamentos de la naturaleza sean apropiadamente valorados y conservados" ${ }^{3}$. De manera específica, esta ley promueve el Principio de Precaución sobre la base de los daños, amenazas y riesgos del medio ambiente, entendido éste como naturaleza.

En la década de los ochentas, específicamente en las Conferencias sobre la protección del Mar del Norte en 1984, 1987 y 1990, las especificaciones del Principio de Precaución tomaban una forma mucho más elaborada en la medida en que las acciones precautorias se llevarían a cabo cuando hubiese razones para suponer un daño cualquiera incluso si faltare prueba científica o relación causal. En el Protocolo de Montreal de 1987, circunscrito a la discusión sobre la capa de ozono, también aparecían las medidas precautorias para controlar de manera equitativa las emisiones globales de sustancias que la debilitasen.

A inicios de la década de los noventas el Principio de Precaución empieza a ser acoplado a la idea de Desarrollo Sostenible, este último entendido como el desarrollo que satisface las necesidades de las generaciones presentes sin comprometer las posibilidades de las generaciones futuras para atender sus propias necesidades ${ }^{4}$. Este acoplamiento se basó específicamente en que el Principio de Precaución era condición necesaria para el logro del desarrollo sostenible.

Así mismo, y con ciertos matices diferenciales, en la Declaración de Río de Janeiro sobre Medio Ambiente y Desarrollo en el año 1992, en la Convención Marco sobre el Cambio Climático de las Naciones Unidas en el año 1992, en la Conferencia Mundial sobre la Ciencia: La Ciencia para el Siglo XXI - Un Nuevo Compromiso, realizada en Budapest el 26 de junio de 1999, en el Protocolo de Cartagena sobre Biodiversidad y Bioseguridad, firmado en Montreal en el año 2000, se hace explícito el Principio de Precaución como instrumento guía para las políticas públicas gubernamentales. Este último protocolo sirve como modelo para la Ley 740 de la República de Colombia de 2002 y el Decreto reglamentario 4525 de 2005 del Ministerio de Agricultura y Desarrollo Rural. También en los Tratados de Maastricht (1992) y Ámsterdam (1994), por los que se constituye la Comunidad Europea y luego Unión Europea, se realza específicamente el Principio de Precaución como el principal principio que deberá orientar la política de la Comunidad en el ámbito del medio ambiente ${ }^{5}$. De hecho, en 1995, Francia incorpora el Principio de 
Precaución a su legislación y desde el año 2000 el Parlamento Europeo enfatiza que el Principio de Precaución constituye un principio esencial de su política. En el caso francés sobresale el hecho de que el Principio de Precaución no solamente se mira desde la gestión del riesgo sino desde las acciones a tomar para evitarlo. El hecho de contemplar de esta manera al riesgo es una de las características esenciales del Principio de Precaución.

Como se ve, durante un proceso de no menos de dos décadas, la discusión del Principio de Precaución tuvo, al menos, tres niveles: pasó de ser una preocupación ambiental basada en la contaminación a una preocupación sobre la base de los costes de daños graves o irreversibles, para luego contemplar el área de la salud pública. Es por estas mismas razones, especificadas en las normativas arriba citadas, que se esperaría que las políticas públicas y las gestiones de las naciones tuvieran en cuenta este Principio tanto en las medidas ambientales como en las políticas de salud pública.

Ahora bien, el Principio de Precaución en la legislación colombiana puede rastrearse desde la ley 99 del año $1993^{6}$, que crea el Ministerio del Medio Ambiente y el Sistema Nacional Ambiental y que, en su artículo 1 numeral 6 reza lo siguiente: "La formulación de las políticas ambientales tendrá en cuenta el resultado del proceso de investigación científica. No obstante, las autoridades ambientales y los particulares darán aplicación al Principio de Precaución conforme al cual, cuando exista peligro de daño grave e irreversible, la falta de certeza científica absoluta no deberá utilizarse como razón para postergar la adopción de medidas eficaces para impedir la degradación del medio ambiente". Después de allí son muchas las leyes de la República y las sentencias de la Corte Constitucional las que se han establecido para que, en términos de las adopciones de la legislación internacional, Colombia implemente el Principio de Precaución. Este Principio es puesto en marcha en el país en aquello que tenga que ver con los costes ambientales, cuando el daño es grave o irreversible, bajo el argumento tanto de la conservación de la diversidad biológica como de la salud humana y el desarrollo sostenible.

Pero entonces ¿cuál es la relación entre medio ambiente y salud, máxime si la salud es entendida desde lo público? De manera evidente, la argumentación tendrá que estar referida a lo que conceptualmente se entiende por salud. Para el caso, es interesante dar un vistazo a la definición que establece, desde 1948, la Organización Mundial de la Salud, la cual queda establecida como: "un estado completo de bienestar físico, mental y social y no simplemente la ausencia de afecciones o enfermedades"7. Se diría entonces que la relación tendría que ser compleja, en la medida en que no podría pensarse el Principio de Precaución sólo sobre la base de los daños ambientales que afecten la salud humana entendida ésta desde las afecciones o enfermedades que le produzca a una población cierto riesgo ambiental. Por lo tanto, inmiscuir dentro del Principio a la salud pública, a la salud colectiva, tendría que implicar tener en cuentas los demás aspectos esenciales del concepto de salud. Algunos autores consideran que las condiciones laborales adecuadas y, por ende, las económicas son parte de la salud; también lo son la equidad, la no discriminación, la igualdad de oportunidades, la participación, el reconocimiento, la libertad, la seguridad, la movilidad, la sustentabilidad ambiental, la confianza en las instituciones, la solidaridad inter e intrageneracional, la información y el respeto, entre otras. Si se toman en cuenta estas condiciones para el bienestar físico, mental y social, entonces la discusión entre Principio de Precaución y salud pública tendría que mirarse, inefablemente, desde el estudio de las políticas públicas en las naciones y desde sus propios regímenes constitutivos. De esta manera, no hay posibilidad de entender la salud sólo de manera individual, ya que si se ahonda en las condiciones de bienestar, efectivamente la salud solamente puede ser colectiva. Sin embargo, la definición arriba nombrada, dada por la OMS, no establece distinción entre las poblaciones humanas, siendo, además, completamente conocido el problema de la inequidad de la calidad de vida en el planeta, como tampoco define a qué se refiere con bienestar físico, mental y social.

Si las políticas de salud pública en Colombia tuviesen en cuenta el Principio de Precaución, como bien lo reza la constitución, tendrían que haber al menos tres cambios en la concepción de las políticas públicas. El primero de ellos sería la inclusión del largo plazo en la evaluación, es decir, que las acciones de salud no dependan de las políticas 
trazadas por planes de gobierno que tienen, por mucho, diez años de duración y que tienen como finalidad mostrar que las metas pudieron cumplirse a través de indicadores. El segundo cambio implica que la salud pública sea entendida como fenómeno planetario, geográfico, histórico y social, es decir, transfronterizo. La tercera es la consideración de las alternativas de acción que incluyan a la inacción como una de ellas. Este cambio pide la definición de alternativas de solución a los problemas existentes y posibles derivados de acciones en salud tanto individuales y colectivas, con miras a tener la posibilidad de elegir aquella alternativa caracterizada por su sustentabilidad ambiental y sus principios de justicia inter e intrageneracional; dentro de las alternativas de acción, la salud pública debe contemplar, de manera comprometida, la alternativa referida a la inacción, especialmente cuando se está hablando de posibles efectos graves, irreversibles o catastróficos.

La discusión con la salud, conectada con las políticas públicas, la salud pública y el Principio de Precaución, toma la variante de la pregunta por la prevención en la medida en que ésta, desde las esferas del saber médico, implica el necesario conocimiento de las certezas científicas tanto para las medidas a tomar como para las políticas y normativas que de allí se deriven. Sin embargo, lejos de concluir que el Principio de Precaución entorpece las medidas de prevención, puede decirse que el Principio de Precaución no va en contra de la prevención, al contrario, ésta es asumida por aquel, ya que ante causas conocidas lo que debe aplicarse es la prevención y cuando no hay conocimiento de las causas, ya sea de tipo parcial o completo, es decir, cuando no se ha logrado establecer una asociación causal entre factores y eventos, el Principio de Precaución se convierte en una eficaz herramienta para la toma de decisiones, ya que, ante la incertidumbre, permite adoptar medidas tendientes a evitar o minimizar los daños a la salud pública o a los ecosistemas ante la sospecha de daños graves e irreversibles. El estudio específico de la situación colombiana implica darle una mirada crítica al Plan Nacional de Salud Pública. Éste tendría como objetivo, según el artículo 33 de la Ley 1122 del 2007 en su capítulo VI sobre la salud pública, "la atención y prevención de los principales factores de riesgo para la salud y la promoción de condiciones y estilos de vida saludables, fortaleciendo la capacidad de la comunidad y la de los diferentes niveles territoriales para actuar". En este objetivo queda claramente establecido que la salud se entiende como carencia de enfermedad y que las acciones gubernamentales tienden a minimizar los riegos para la salud así entendida. Queda de manifiesto entonces que la noción de bienestar físico y social no está ni explícita ni implícitamente contemplado en la legislación en salud de Colombia.

Este mismo artículo incluye un listado de los aspectos que debe incluir el Plan, en los que se hace evidente la inexistencia del Principio de Precaución y la reducida mirada de la salud, en tanto la legislación está basada en el aseguramiento -como bien lo dictamina la Ley $100-$ y no en el bienestar colectivo. De hecho, en este mismo listado no hay una relación evidente o explícita entre la salud pública y el compromiso de la calidad del medio ambiente como elemento de trascendental importancia en el logro del bienestar de la comunidad y la disminución de la morbilidad y la mortalidad. Esto quiere decir, al menos, que la otrora relación entre salud pública y medio ambiente con relación al Principio de Precaución queda completamente eliminada en la legislación colombiana. La norma que determina la obligatoriedad de elaboración del Plan Nacional de Salud Pública considera que éste debe plantearse para cada cuatrienio, con lo cual la posibilidad de planeación e intervención en el largo plazo se hace muy difícil. También es evidente la ausencia de preocupación por los fenómenos internacionales y transfronterizos que podrían llegar a ser de interés para la salud pública de la región.

En cuanto a los principios, el Plan Nacional de Salud Pública asume los siguientes: universalidad, equidad, calidad, eficiencia, responsabilidad, respeto por la diversidad cultural y étnica, participación social e intersectorialidad. Estos principios se han establecido, desde 1991, como necesarios para el funcionamiento de las políticas públicas, como puede leerse en la Constitución Política de Colombia y, sin duda, no pueden dejarse de lado en las planeaciones gubernamentales de turno. No obstante, y como se dijo anteriormente, sí se ha dejado de lado en las planeaciones referidas a la salud pública el Principio de Precaución; esto se evidencia en las líneas de la política del Plan Nacional de Salud Pública, las cuales son las siguientes: 1) la promoción de la salud y la calidad de vida; 2) la prevención de los riesgos; 3) la recuperación y superación de los daños en la salud, 4) la vigilancia en salud y gestión del conocimiento; y 5) la gestión 
integral para el desarrollo operativo y funcional del Plan. Aunque cada uno de los principios y cada una de las líneas de acción tienen una explicación y un sustento, asombra el hecho de que se omitan asuntos esenciales de esta temática. Un buen ejemplo es la poca claridad que se tiene en el Plan acerca de la definición de calidad de vida, en donde ésta queda en las manos de las percepciones individuales con relación a las expectativas, metas, normas y preocupaciones. No existe, como se ve, una mirada que vaya más allá de lo puramente perceptivo individual, lo que vuelve a entorpecer la mirada colectiva y pública de la salud como bienestar social con todas las características que implica. De hecho las percepciones subjetivas pueden no incorporar problemáticas tales como los impactos ambientales derivados de megaproyectos, en la medida en que los sujetos podrían percibir un incremento económico sin que por ello las consecuencias de daños graves o irreversibles sean tomadas en cuenta.

Las políticas públicas en salud del actual gobierno colombiano, referidas a los riesgos, parecieran se concentran en dos líneas: la prohibición y la obediencia a las medidas preventivas, lo que quiere decir que la responsabilidad gubernamental frente al riesgo es casi nula y la información sobre la base de los riesgos es completamente selectiva. Como ejemplo de ello están las campañas de no conducir embriagado y la inexistencia de campañas de no consumir alimentos transgénicos.

Muchos son los decretos, las leyes y las características de los planes nacionales que refieren específicamente a la salud y a su legislación. La Constitución Política de Colombia refiere específicamente el Principio de Precaución. No obstante, en el Plan Nacional de Salud Pública no se menciona el Principio de Precaución como orientador de la política pública y tampoco se evidencia la apropiación del mismo, entre otras cosas, porque no se contemplan aspectos relacionados con la magnitud de los eventos a los cuales se debería aplicar, no se mencionan propuestas a largo plazo, está basado en certidumbres científicas, se circunscribe a circunstancias nacionales, se aplica un concepto de riesgo restringido, opta por la acepción simple de la salud, se compromete con la definición unidimensional de ambiente y está basado en actividades de prevención.

Tenemos entonces que la importancia del Principio de Precaución en las políticas públicas tanto relacionas con el medio ambiente como con la salud humana, es innegable. Los organismos internacionales han estado a tono con esta importancia y para ello han establecido legislaciones y decretos que implementan y dan cabida a tal Principio. Las preocupaciones acerca del adelantado desarrollo tecnológico y las ahora concretas imposibilidades de la certeza científica absoluta, deben hacer parte de los fundamentos de cualquier diseño, planeación y ejecución de una política pública nacional y debe tener, en todos los casos, una mirada transfronteriza. Sin lugar a dudas, la investigación médica debe cruzar los límites de lo que estrictamente contiene su disciplina para poder entablar una discusión con las normativas nacionales e internacionales que respectan a la salud, como también es necesario que la investigación médica devele problemas y sugiera aportes a las conceptualizaciones que se hacen en tales leyes a propósito del tema de la salud humana.

Para terminar y como conclusión de este artículo, se transcriben de manera literal las conclusiones y las recomendaciones que el informe final de la investigación, que aquí se resume, establece:

El Principio de Precaución incluye la prevención y la supera; se convierte así en un instrumento útil de gestión y decisión cuando la ciencia no suministra, con su metodología, la posibilidad de evitar el potencial daño del medio ambiente y la salud pública por sus propios desarrollos.

Es posible determinar la apropiación del Principio de Precaución en las políticas públicas sanitarias al tener en cuenta: a) que la fundamentación teórica de cada riesgo incluya su definición, análisis, gestión y comunicación; b) que se haga evidente la participación de todos los intervinientes en los cuatro elementos mencionados; c) que el análisis del riesgo no sólo esté basado en la certidumbre científica absoluta; d) que en la gestión del riesgo se plantee la posibilidad de actuar o de no actuar ante la sospecha de daños graves, irreversibles o catastróficos; e) que exista planeación a largo plazo de tal manera que se 
incluya la perspectiva de las generaciones futuras y de la sustentabilidad; f) que esté abierta a los avances de la ciencia de tal manera que su revisabilidad conduzca a la realización de ajustes oportunos; y g) que haya una definición jurídica de incertidumbre.

El Principio de Precaución sí está consagrado en la legislación colombiana y se encuentra bien sustentado en las diferentes Sentencias de la Corte Constitucional que se han emitido al respecto; además, la legislación colombiana asume los principales acuerdos internacionales vinculantes sobre la temática ambiental y de salud pública.

En el Plan Nacional de Salud Pública no se menciona el Principio de Precaución como orientador de la política pública y tampoco se evidencia la apropiación del mismo, entre otras cosas, porque no se contemplan aspectos relacionados con la magnitud de los eventos a los cuales se debería aplicar, no se mencionan propuestas a largo plazo, está basado en certidumbres científicas, se circunscribe a circunstancias nacionales, se aplica un concepto de riesgo restringido, opta por la acepción simple de la salud, se compromete con la definición unidimensional de ambiente y está basado en actividades de prevención.

En toda escuela de salud pública, así como en los procesos formativos de todos los pregrados y postgrados que de una u otra manera se relacionen con el abordaje de la enfermedad, se debe tener como temática obligatoria el estudio del Principio de Precaución y como competencia específica la capacidad de analizar críticamente las políticas públicas sanitarias para superar el simple aprendizaje de su aplicación.

Las políticas públicas sanitarias deben tener elementos para el largo plazo, pues su definición como parte de los planes de gobierno, con una vigencia de cuatro años, sólo puede atender fenómenos presentes y de rápida intervención que permitan generar indicadores relacionados con la buena gestión.

Se hace necesario que las políticas públicas sanitarias contengan temáticas globales relacionadas con el medio ambiente, pues está claramente demostrada su estrecha relación con la salud pública; así, las políticas públicas sanitarias deben tener como visión la permanencia de la humanidad en el planeta en condiciones dignas o, al menos, con las mismas con que contamos hoy en día.

La definición y análisis de riesgo no debe ser realizada sólo por los expertos, debe incluir a todos los intervinientes: comunidad, medio de comunicación, clero, minorías étnicas, investigadores independientes, observadores internacionales, etc.

Desde la academia se debe propender porque el Principio de Precaución se incluya en todas las políticas públicas referidas a la salud, tal y como está establecido por la ley; así mismo porque se vigilen las amenazas que pueden causar daños graves e irreversibles al ecosistema y a la salud pública sin esperar legislaciones y normativas específicas.

\section{BIBLIOGRAFÍA}

- Escajedo Sandepifanio, L. Principio de Precaución y riesgos ambientales. En: Romeo Casabona, CM (ed). Principio de precaución, biotecnología y derecho. España: Fundación BBVA; 2004. p. 149-218.

- Vorsongende Umweltpolitik, Gobierno Federal Alemán, 1976.

- Organización de las Naciones Unidas. Nuestro Futuro Común. Comisión Mundial del Medio Ambiente y del desarrollo. Colombia: Alianza; 1988.

- Tratado constitutivo. Artículo 174. Título XIX. Comunidad Económica Europea.

- Ley 99/1993 de 22 de Diciembre. Por la cual se crea el Ministerio del Medio Ambiente, se reordena el Sector Público encargado de la Gestión y Conservación del Medio Ambiente y los Recursos Naturales Renovables, se organiza el Sistema Nacional Ambiental, SINA y se dictan otras disposiciones. Congreso de la República de Colombia. 
- Organización Mundial de la Salud. Constitución de la Organización Mundial de la Salud. Documentos básicos, suplemento de la 45a edición, octubre de 2006.

- Plan Nacional de Salud Pública 2007-2010. Ministerio de la Protección Social. República de Colombia.

- Ley 1122/2007 de 9 de Enero. Por la cual se hacen algunas modificaciones en el Sistema General de Seguridad Social en Salud y se dictan otras disposiciones. Congreso de la República de Colombia.

- Ley 100/1993 de 23 de Diciembre. Por la cual se crea el sistema de seguridad social integral y se dictan otras disposiciones. Congreso de la República de Colombia.

- Sentencia C-293/02. 23 de Abril. Referencia: expediente D-3748. Corte Constitucional. Colombia.

- Ley 740/02 de 24 de Mayo. Por medio de la cual se aprueba el "Protocolo de Cartagena sobre Seguridad de la Biotecnología del Convenio sobre la Diversidad Biológica", hecho en Montreal, el veintinueve (29) de enero de dos mil (2000). Congreso de la República de Colombia.

- Ley 164/ 94 de 27 de Octubre. Por la cual se aprueba la "Convención Marco de las Naciones Unidas sobre el Cambio Climático", hecha en Nueva York el 9 de mayo de 1992. Congreso de la República de Colombia.

- Ley 629/00 de 27 de Diciembre. Por medio de la cual se aprueba el "Protocolo de Kyoto de la Convención Marco de las Naciones Unidas sobre el Cambio Climático", hecho en Kyoto el 11 de diciembre de 1997.

- Decreto reglamentario 4525 de 2005. Ministerio de Agricultura y desarrollo rural. Colombia.

- Resolución 0425 de febrero de 2008. Ministerio de la Protección Social. Colombia.

- Decreto 1950 de 1964. Presidencia de la República. Colombia.

- Decreto 677 de 1995. Presidencia de la república. Colombia.

- Resolución COM 20030644 (04). Comisión Europea. Unión Europea. Sentencia TS $2^{\circ}$, S 15-12-2000. Tribunal Supremo. España.

- Loi Barnier del 2 de febrero de 1995. Francia.

- Álavarez Alva R. Salud pública y medicina preventiva. México: Manual moderno; 2002.

- Amaya Navas OD. La constitución ecológica de Colombia. Colombia: Universidad Externado de Colombia; 2002.

- Beck U. La sociedad del riesgo. Hacia una nueva modernidad. Barcelona: Paidos; 1998.

- $\quad$---------. Políticas ecológicas en la edad del riesgo. Barcelona: El roure; 1998.

- $\quad$ Blum, H.L. Planning for Health. New York: Human sciences press; 1981.

- Borrero Navia JM. Los derechos ambientales. Una visión desde el Sur. Feriva: Cali; 1994

- Bronfman M, Langer A, Trostle J. De la investigación en salud a la política: la difícil traducción. México: Manual moderno; 2000.

- Dobson A. Pensamiento político verde. Una nueva ideología para el siglo XXI. Barcelona: Paidos; 1997.

- $\quad$---------. Pensamiento verde: una antología. Valladolid: Trotta; 1999.

- Frenk J. La salud de la población. Hacia una nueva salud pública. México: FCE; 2003.

- García A. Negociar el riesgo. Una propuesta para la gestión de riesgos en sistemas tecnológicos complejos. [Tesis doctoral]. Barcelona; 2004

- Gómez Heras JM. Ética del medio ambiente. Problema, perspectiva e historia. Madrid: Tecnos; 1997.

- López Cerezo JA, Luján JL. Ciencia y política del riesgo. Madrid: Alianza; 2000

- Meadows DH. Los límites del crecimiento: informe al Club de Roma sobre el predicamento de la Humanidad. México: FCE; 1972.

- Miller H, Conko G. Precaution without principle. En: Nature biotechnology. vol 19; abril de 2001

- Organización de las Naciones Unidas. Convención Marco sobre el Cambio Climático de las Naciones Unidas; 2002. 
- Organización de las Naciones Unidas. Convenio de Estocolmo sobre Contaminantes Orgánicos Persistentes; 2002.

- Organización de las Naciones Unidas. Declaración de Río de Janeiro sobre Medio Ambiente y Desarrollo. Brasil; 1992.

- Organización de las Naciones Unidas. Informe sobre desarrollo humano; 20072008.

- Organización de las Naciones Unidas. Informe de la Conferencia de las Naciones Unidas sobre Medio Ambiente Humano; 1972.

- Organización de las Naciones Unidas. Protocolo de Montreal relativo a las sustancias que agotan la capa de ozono; 1987

- Organización de las Naciones Unidas. Convenio de Estocolmo sobre contaminantes orgánicos persistentes; 2001.

- Organización de las Naciones Unidas para la Educación, la Ciencia y la Cultura. Conferencia Mundial sobre la Ciencia. Budapest, 1999.

- $\quad$ Prigogine I. El fin de las certidumbres. España: Taurus; 1997.

- Riechmann J, Tickner J. El principio de precaución. Barcelona: Icaria; 2002.

- Rifkin J. El siglo de la biotecnología. El comercio genético y el nacimiento de un mundo feliz. Barcelona: Paidos; 1999.

- Roa Rojas H, Roa Días C. Régimen ambiental. Colombia: Ecoe; 2007.

- Samaja J. Epistemología de la salud. Argentina: Lugar; 2004.

- Secretaría del convenio sobre la diversidad biológica (2000). Protocolo de Cartagena sobre Seguridad de la Biotecnología del Convenio sobre la Diversidad Biológica: texto y anexos. Montreal: Secretaría del Convenio sobre la Diversidad Biológica.

- Sousa B. El foro social mundial. En: Revista el viejo topo: № 240; Enero de 2008.

- $\quad-------$ La universidad en el siglo XXI. La Habana: Casa de las Américas; 2006.

- Vega Franco L. La salud en el contexto de la nueva salud pública. México: Manual moderno; 2000.

\section{NOTAS}

1. La metodología que aquí se transcribe es la correspondiente al informe final de la investigación“Apropiación del Principio de Precaución en las políticas públicas sanitarias: caso Plan Nacional de Salud Pública de Colombia".

2. Escajedo Sanepifanio, L. Principio de precaución y riesgos ambientales. En: Romeo Casabona, CM (ed). Principio de precaución, biotecnología y derecho. España: Fundación BBVA, 2004. p. 189

3. Vorsongende Umweltpolitik, Gobierno Federal Alemán, 1976.

4. Cfr. Organización de las Naciones Unidas. Nuestro Futuro Común. Comisión Mundial del Medio Ambiente y del desarrollo. Colombia: Alianza; 1988.

\section{Tratado constitutivo. Artículo 174. Título XIX. Comunidad Económica Europea.}

6. Ley 99/1993 de 22 de Diciembre. Por la cual se crea el Ministerio del Medio Ambiente, se reordena el Sector Público encargado de la Gestión y Conservación del Medio Ambiente y los Recursos Naturales Renovables, se organiza el Sistema Nacional Ambiental, SINA y se dictan otras disposiciones. Congreso de la República de Colombia.

7. Organización Mundial de la Salud. Constitución de la Organización Mundial de la Salud. Documentos básicos, suplemento de la 45a edición, octubre de 2006. La Constitución fue adoptada por la Conferencia Sanitaria Internacional, celebrada en Nueva York del 19 de junio al 22 de julio de 1946, firmada el 22 de julio de 1946 y entró en vigor el 7 de abril de 1948. 